\title{
Transatlantica
}

Revue d'études américaines. American Studies Journal

$2 \mid 2020$

Places and Cultures of Capitalism: Histories from the

Grassroots

\section{La démocratie américaine à l'épreuve de la présidence Trump}

Université de Bordeaux, 22 janvier 2021

\section{Clémence Faure}

\section{OpenEdition}

\section{Journals}

Édition électronique

URL : https://journals.openedition.org/transatlantica/16268

DOI : 10.4000/transatlantica. 16268

ISSN : 1765-2766

Éditeur

Association française d'Etudes Américaines (AFEA)

\section{Référence électronique}

Clémence Faure, "La démocratie américaine à l'épreuve de la présidence Trump », Transatlantica [En ligne], 2 | 2020, mis en ligne le 10 mars 2021, consulté le 01 février 2023. URL : http:// journals.openedition.org/transatlantica/16268; DOI : https://doi.org/10.4000/transatlantica.16268

Ce document a été généré automatiquement le 1 février 2023.

\section{(c) (i) $\ominus$}

Creative Commons - Attribution - Pas d'Utilisation Commerciale - Pas de Modification 4.0 International - CC BY-NC-ND 4.0

https://creativecommons.org/licenses/by-nc-nd/4.0/ 


\section{La démocratie américaine à l'épreuve de la présidence Trump}

Université de Bordeaux, 22 janvier 2021

\section{Clémence Faure}

\section{NOTE DE L'AUTEUR}

Lien vers le programme du colloque : https://irm.u-bordeaux.fr/Actualites/22-JanvierLa-democratie-americaine-a-l-epreuve-de-la-presidence-Trump

1 Le colloque "La démocratie à l'épreuve de la présidence Trump », organisé par David Diallo, Éric Rouby et Adrien Schu, s'est tenu le 22 janvier 2021 à l'université de Bordeaux. Cette manifestation, soutenue par l'Institut des Amériques, le laboratoire Climas (université Bordeaux Montaigne) et l'Institut de Recherche Montesquieu (université de Bordeaux) visait, dans le contexte mouvementé de l'après-présidentielle et seulement deux jours après l'investiture de Joe Biden, à faire un premier bilan de la présidence Trump dans ses rapports aux institutions, aux normes et aux mouvements qui structurent la démocratie américaine et permettent son fonctionnement. Affichant une ambition résolument pluridisciplinaire et faisant dialoguer, dans cette perspective, chercheurs et chercheuses en civilisation américaine, sociologie et science politique, ce colloque s'est donné pour objectif de questionner l'ère Trump au travers de trois problématiques: la construction trumpiste des «ennemis » de l'intérieur; les enjeux électoraux et partisans; la structuration et l'organisation au sein de la société étasunienne contemporaine des divisions et des contestations.

\section{Donald Trump et les « ennemis » de l'intérieur}

2 Le colloque s'est ouvert avec l'introduction d'Adrien Schu (université de Bordeaux), introduction ambitionnant de cadrer la réflexion collective sur le rapport du trumpisme à la démocratie au prisme de la tension entre continuité et rupture : alors 
même que les commentateurs ont tendance à faire de l'ère Trump une parenthèse politique caractérisée par une remise en cause des principes et des valeurs de la démocratie étasunienne, l'effritement de celle-ci n'aurait-il pas commencé avant Trump? Ce dernier est-il une anomalie politique ou le symptôme d'une crise plus profonde de la société démocratique américaine?

3 Nourri de ces questionnements, le premier panel « Donald Trump et les "ennemis" de l'intérieur » a débuté avec l'intervention de Sébastien Mort (université de Lorraine), venu interroger les diverses modalités et les divers objectifs de la stratégie trumpiste d'intimidation des médias d'information. Associant spectacle politique, menaces de représailles institutionnelles, humiliations publiques, remises en cause de l'intégrité des journalistes voire encouragement des partisans pro-Trump à participer activement à cette campagne de délégitimation, cette stratégie confine à l'intimidation performative. En cela, si elle reprend des éléments bien connus des diverses critiques contemporaines adressées aux médias dominants, il reste qu'elle innove dans ses objectifs : il ne s'agit pas pour Donald Trump de manipuler les médias d'information américains ou de les empêcher de fonctionner mais bien de faire le show pour renforcer le lien avec ses soutiens via la désignation d'un ennemi commun. Si ces intimidations et ces menaces s'avèrent ainsi de pure forme, sans effet institutionnel ou juridique concret, elles ont pour conséquence non seulement d'affaiblir la crédibilité des médias d'information dominants mais également de les obliger à adopter une même posture défensive, laquelle les constitue, en dépit de leur diversité, en un ensemble uniforme qualifié par Donald Trump et ses soutiens de médias « mainstream ».

4 La contribution de Gildas Le Voguer (université Rennes 2) s'est concentrée quant à elle sur les enjeux du renseignement sous l'ère Trump au travers de l'analyse du Russiagate. L'affaire russe doit, avant tout, être replacée dans le contexte d'une dégradation progressive, à partir du début des années 2000, des relations américano-russes. La guerre en Irak, l'intervention en Lybie, les manifestations des opposants russes en 2011 et les Panama Papers sont les étapes principales de l'augmentation des tensions entre les deux pays. Elles viennent éclairer la volonté de la Russie de mettre en place une stratégie d'espionnage offensive, stratégie qui mobilise alors des moyens anciens mais dont l'originalité réside dans le recours aux nouvelles technologies. Compte tenu de ce contexte, l'incapacité de la CIA à déjouer l'espionnage russe procède d'un manque d'anticipation qui tire lui-même son origine d'un problème d'orientation: la priorité étant, depuis le 11 septembre 2001, la lutte contre le terrorisme, la plus grande partie des moyens du renseignement lui est allouée. De plus, la Russie étant considérée, dans ce combat, comme un partenaire, la surveillance de ses activités n'est pas un objectif prioritaire. Depuis 2001, les services de renseignements sont, en outre, particulièrement affaiblis, et cela en raison de nombreux scandales qui les ont forcés à se réorganiser parfois au détriment de leur mission première de renseignement.

5 Ce premier panel s'est clôturé avec l'intervention d'Éric Rouby (université de Bordeaux) qui s'est attaché à questionner les ressorts discursifs de la construction des «ennemis de l'intérieur» aux États-Unis, ainsi que ses causes et ses conséquences extra-idéologiques. Le discours de l'ennemi est tout entier acte de langage et a pour principal objectif de constituer un groupe ou un individu comme interlocuteur illégitime. Aux États-Unis, les divers discours de l'ennemi recourent ainsi à trois types de rhétorique de délégitimation: est ennemi celui qui s'écarte des traditions dites américaines ; celui qui est désigné comme juridiquement étranger ou comme étant à la 
solde de l'étranger; celui qui est identifié comme faisant partie du groupe des " privilégiés " par opposition à celui des " laissés pour compte ». Ces trois rhétoriques ont pour point commun d'user de termes flous et de s'appuyer sur une sémantique guerrière visant à officialiser l'existence d'une culture war en cours. La prolifération, aux États-Unis, de ces discours de l'ennemi est corrélée à une polarisation des positionnements politiques et a pour conséquence essentielle de remettre en cause le fonctionnement par compromis sur lequel se fonde la démocratie américaine : parce que ces discours transforment l'adversaire en ennemi politique inféodé aux intérêts du camp adverse, il n'est dès lors plus possible non seulement de travailler avec lui mais également de mettre au jour et de servir avec lui l'intérêt général.

\section{Trump ou la démocratie?}

6 Le deuxième panel «Trump ou la démocratie? » a été introduit par Éric Dubesset (université de Bordeaux). Celui-ci a voulu attirer l'attention des participants sur les enjeux épistémologiques de cette manifestation scientifique. En particulier, l'analyse de l'opposition radicale qu'il y aurait entre régime démocratique et Trump nous offre la possibilité de réfléchir au concept même de démocratie. In fine, la question essentielle serait moins celle des éléments attestant cette antinomie que de savoir ce que la situation américaine nous dit de la démocratie aujourd'hui.

7 Les deux premières contributions, "Le droit de vote à l'épreuve de la présidence Trump » et «Ne rien concéder : une fin de mandat entre chaos et continuité » sont chacune venues nous rappeler que la question des conditions d'accès au vote était déjà un enjeu électoral avant la présidence Trump.

8 Comme l'a tout d'abord expliqué Olivier Richomme (université Lumière Lyon 2), cet enjeu dérive du traumatisme institutionnel créé par les élections de 2000 qui ont vu George W. Bush l'emporter face à Al Gore alors même que ce dernier le devançait dans le vote populaire. Si la loi HAVA de 2002 a tenté de résoudre ces écueils institutionnels, elle n'a pas empêché que l'accès au vote devienne un outil électoral stratégique pouvant servir diversement les intérêts des deux grands partis américains. Ainsi, alors que les Démocrates cherchent à le faciliter - les électeurs ayant plus de difficultés à voter leur étant a priori plus favorables - les Républicains tentent quant à eux soit de le restreindre soit d'en contester a posteriori la procédure via la dénonciation de fraudes électorales. Le fait que la problématique de la fraude ait été placée, par le camp trumpiste, au centre du débat public dans la période post-présidentielle n'est donc pas un événement électoral inédit: il s'agit d'une tactique qui fait partie intégrante de la stratégie électorale républicaine depuis les années 2000, et dont le parti use en fonction des rapports de force partisans. Dans cette perspective, il n'est pas non plus étonnant que le vote par correspondance, censé améliorer l'accès aux urnes, se soit constitué en enjeu électoral fortement clivant. La crise du Covid n'a fait qu'en accentuer le caractère stratégique. Reste que cette élection aura permis de mettre en évidence les risques que peuvent faire courir à la démocratie américaine la politisation du droit de vote et son érection en enjeu partisan.

9 Dans une perspective complémentaire, Marion Douzou (université Lumière Lyon 2) a insisté sur le rôle joué, au sein du Parti républicain, par la nébuleuse conservatrice dans cette politisation du droit de vote. Ce rôle atteste là encore que le positionnement de Trump sur cet enjeu doit être analysé comme un élément de continuité d'une stratégie 
élaborée à la suite du traumatisme électoral de 2000. Le camp conservateur s'est ainsi employé, dès cette période, à faire de la fraude un problème public majeur. Cette problématisation s'est appuyée, dans divers États, sur le vote de lois visant à lutter contre la fraude, conditionnant notamment la participation électorale à la présentation d'une pièce d'identité - autant de mesures qui ont de fait rendu plus contraignant l'accès aux urnes pour certains segments de l'électorat davantage enclins à voter Démocrate. Elle a également été permise par la mobilisation des militants et des sympathisants républicains via la diffusion de publicités anti-fraude, de brochures explicatives médiatisant les rares cas avérés de fraude ou encore l'organisation de formations censées permettre aux membres du parti de la contrecarrer. La présence au sein de l'entourage politique de Donald Trump de proches voire de membres du Tea Party, et cela à des postes clés, permet aussi de replacer les positionnements du Président à l'égard du droit de vote dans une certaine continuité stratégique ainsi qu'idéologique du mouvement conservateur et manifeste en cela une certaine pérennité des jeux de pouvoirs au sein du Parti républicain.

C'est à ces jeux de pouvoirs intrapartisans que s'est ensuite intéressé François Vergniolle de Chantal (université de Paris) dans sa contribution «Le Parti républicain peut-il survivre à Trump ?». La défaite du Président sortant plonge le parti dans une crise bien plus profonde que celle qu'il a connue après l'ère W. Bush. La fragmentation des élites, observable en son sein depuis la fin des années 2000, s'est sensiblement accentuée durant la présidence Trump. Cette cristallisation des oppositions s'est traduite, pour ces élites, par une perte du contrôle de la sélection des candidats, celle-ci s'opérant désormais sur des critères charismatiques qui nécessitent toujours davantage l'appui de la base partisane. Le parti se trouve en outre confronté à une crise idéologique qui manifeste un certain épuisement de la révolution conservatrice des années 1980. Le trumpisme est le symptôme de cette érosion et participe à rendre moins lisible la marque républicaine. Peu consistant idéologiquement, il se caractérise avant tout par une personnalisation des enjeux et des rivalités qu'il simplifie et qu'il rend accessibles au plus grand nombre. Enfin, le très fort soutien des électeurs républicains, dont Trump continue à bénéficier, s'est matérialisé non seulement dans les urnes - il a réalisé malgré sa défaite le meilleur résultat électoral de l'histoire du parti - mais également par l'augmentation significative du poids des petites donations dans le budget de campagne. Compte tenu des intérêts électoraux en jeu, l'héritage du trumpisme s'avère donc difficile à solder pour le Parti républicain et met in fine son unité en sursis.

\section{Dynamiques, divisions et contestations au sein de la société américaine}

11 C'est David Diallo (université Bordeaux Montaigne) qui a présenté le troisième et dernier panel de cette rencontre portant sur la configuration des contestations et la structuration des divisions au sein de la société américaine. Il a notamment rappelé que le mandat de Donald Trump s'est accompagné d'une augmentation sensible des tensions sociales et identitaires que symbolisent notamment la médiatisation des mouvements \#MeToo en 2017 ou Black Lives Matter en 2020. Cette augmentation nous invite alors à nous interroger sur les dynamiques qui ont fait du genre et de la race des 
catégories explicatives et opérantes permettant d'éclairer l'organisation institutionnalisée des rapports sociaux.

12 La présidence Trump incarne de manière évidente une contre-réaction à la visibilisation des mouvements féministes. Il reste néanmoins à trouver un modèle capable d'en restituer les dynamiques. C'est à cette question que s'est consacré David Bertrand (université de Bordeaux). Parmi les multiples paradigmes mobilisables pour comprendre la configuration de la cause des femmes, celui du «backlash», tel que conceptualisé par Mansbridge et Shames ${ }^{1}$, connaît un certain succès médiatique et académique. Ce modèle comporte pourtant des écueils qui le rendent peu opérant. En particulier, la mobilisation du concept de pouvoir sur lequel il se fonde parait bien souvent superfétatoire. Ainsi, le sentiment de perte de pouvoir qui motiverait le backlash échoue à éclairer les dynamiques en jeu. Ce modèle conduit, de plus, à adopter une lecture binaire des oppositions et invisibilise dès lors les alliances qui peuvent se former sur certains thèmes entre mouvement et contre-mouvement. Surtout, il masque la pluralité interne du féminisme et ne permet pas de mettre en lumière les divers espaces de lutte (réels et virtuels) investis par les femmes. L'hypothèse de l'apparition aux États-Unis d'une quatrième vague féministe, corroborée notamment par le renouvellement générationnel des militantes et le recours aux réseaux sociaux, permet de pallier ces limites et d'éclairer davantage la multiplication des contre-mouvements conservateurs et finalement les positionnements antiféministes de Trump et de son administration.

Enfin, Damien Simonneau (Collège de France) s'est intéressé au rôle de l'enjeu migratoire dans la formation du choix électoral au sein de l'électorat hispanique. L'Arizona est, de ce point de vue, intéressant à étudier. Cet État a connu des évolutions socio-démographiques importantes : à côté de l'attrait qu'il suscite auprès des jeunes diplômés, sa population est composée pour un tiers d'Hispaniques qui sont par ailleurs plus jeunes, plus pauvres et moins éduqués que les non hispaniques. Il est, en outre, le fer de lance, depuis les années 2000 , de la politique sécuritaire républicaine en matière migratoire, politique reprise au niveau national dans les années 2010 et qui a inspiré les choix opérés sur ce thème par l'administration Trump. L'Arizona est aussi au centre de la bataille partisane entre Démocrates et Républicains, bataille que matérialise le faible écart de voix avec lequel Joe Biden l'a finalement emporté dans cet État traditionnellement rouge. Compte tenu de ce contexte, la forte et pérenne mobilisation de citoyens et d'associations hispaniques contre la politique migratoire arizonienne éclaire le basculement électoral de 2020. Électoralisant l'enjeu migratoire, ces groupes ont fait du combat contre la réélection des Républicains, de l'inscription sur les listes électorales, du soutien officiel à des candidats démocrates ou encore de l'incitation au vote, autant de moyens de lutte contre la répression de l'immigration. Cette fusion des enjeux viendrait alors éclairer la corrélation, dans cet État, entre augmentation du nombre d'inscrits, forte participation et croissance sensible du vote démocrate.

Ce colloque bordelais a ainsi permis d'effectuer un premier bilan de la présidence Trump. La diversité des thèmes abordés témoigne de l'ampleur des bouleversements politiques dont le trumpisme est sinon la cause du moins le symptôme. Nous regrettons toutefois que certains phénomènes soient restés dans l'angle mort de cette manifestation. Ainsi aurait-on apprécié que l'analyse théorique de la configuration de l'espace de la cause des femmes fût complétée par une étude davantage sociohistorique. Cette étude aurait permis non seulement de mettre en évidence les 
dynamiques culturelles, sociales et électorales à l'œuvre dans la problématisation de cette cause mais également d'éclairer les événements et le contexte politique sur lesquels cette problématisation s'est appuyée. De même, les ressorts du soutien électoral à Trump n'ont pas été vraiment questionnés. Si les motivations à l'œuvre dans le vote hispanique pro-Trump ont été largement déblayées dans les débats ayant suivi les interventions, les variables socio-économique et socio-géographique n'ont pas été discutées. Dans cette perspective, qu'aurait pu nous dire du trumpisme mais aussi du rapport de l'électorat à la démocratie, la sociologie des électeurs républicains de 2016 et 2020 ? Malgré ces quelques remarques, les analyses présentées dans ce colloque (mouvements sociaux, renseignement, enjeux électoraux et partisans, évolutions intrapartisanes, polarisation des positionnements politiques, etc.) ont démontré de manière convaincante que le trumpisme, loin d'être une parenthèse politique, devait être replacé dans la continuité d'une période de crise qui débute au début des années 2000. Au-delà et quand bien même la question de la base électorale et des contours du soutien populaire à Trump n'a été qu'esquissée, cette manifestation est venue nous rappeler qu'à l'arrière-plan de la relation du trumpisme à la démocratie, c'est bien celle unissant les représentants aux représentés dont il est question. Ainsi, si la société américaine est confrontée à des enjeux qui lui sont spécifiques, cette manifestation tend à rapprocher la crise démocratique à laquelle elle est en proie de celle que connaît depuis vingt ans l'ensemble des démocraties occidentales. Ce colloque incite dès lors à poursuivre la discussion et à assister, pour ce faire, à la prochaine rencontre dont les organisateurs ont annoncé qu'elle aurait pour thème la politique étrangère sous l'ère Trump.

\section{NOTES}

1. MANSBRIDGE Jane et SHAMES Shauna. « Toward a Theory of Backlash: Dynamic Resistance and the Central Role of Power ». Politics \& Gender Journal, n4, 2008, p. 623-634.

\section{AUTEUR \\ CLÉMENCE FAURE}

Université de Bordeaux 